\title{
Systemic Administration of Granulocyte Colony-Stimulating Factor for Osteochondral Defect Repair in a Rat Experimental Model
}

\author{
Tadashi Okano', Hisashi Mera², Maki Itokazu',2, Takahiro Okabe ${ }^{3}$, Tatsuya Koike ${ }^{4}$, \\ Hiroaki Nakamura', and Shigeyuki Wakitani ${ }^{2}$
}

\begin{abstract}
Objective. The objective of this study was to assess the effect of granulocyte colony-stimulating factor (G-CSF) on osteochondral defect repair in the rat knee. Design. Twenty-six 12-week-old male Lewis rats were randomly divided into 2 groups. From day 0 to day 4, the G-CSF group received glycosylated G-CSF, and the control group received phosphatebuffered saline. A $1.5-\mathrm{mm}$ diameter and I.0-mm deep osteochondral defect was introduced in the patellar groove of the bilateral femur in all rats on day 4 . The peripheral blood nucleated cells were counted for 14 days from the first day of injection, the appearance of the cartilage repair was observed histologically and macroscopically for $2,4,8,12$, and 24 weeks after surgery. Results. The number of peripheral blood leukocytes increased 3 days and returned to normal levels 7 days after the first injection. Compared with the control group, the G-CSF group had more fibrous and/or bony tissue at earlier points in time. The tissue repair rate, which is defined as the percentage of repaired osteochondral defects, was significantly higher in the G-CSF group 4 weeks after surgery. However, there were no significant differences in the cartilage repair rate and the modified Wakitani score between the 2 groups at each time point. Conclusions. The defect filling was significantly better in the G-CSF group in the early phases. Our findings suggest that G-CSF may promote the repair of osteochondral defects by mediating an increase in the number of peripheral blood nucleated cells.
\end{abstract}

\section{Keywords}

osteochondral defect, bone repair, cartilage repair, granulocyte colony-stimulating factor (G-CSF), peripheral blood

\section{Introduction}

Osteochondral defects are quite common and are notably reported in approximately $20 \%$ of all arthroscopic procedures. ${ }^{1,2}$ Moreover, it has been reported that up to $43 \%$ of healthy subjects even without a family history of osteoarthritis (OA) would have a knee cartilage lesion detected by MRI. ${ }^{3}$ Such lesions are clinically important because they could cause pain, locking of the joint, and/or other symptoms leading to the subject's disability. They might progress further cartilage loss and result in severe OA over an extended period. ${ }^{4}$

The most significant problem associated with osteochondral defects is the limited capacity for repair. ${ }^{5}$ It is known that there are critical sizes for repairable defects, depending on each species. ${ }^{6,7}$ In other words, full repair would not be expected for any defect larger than the critical size. The reasons of this limited repair capacity are not completely understood; however, it is thought that an insufficient supply of progenitor cells and/or growth factors due to avascularity in the lesion of articular cartilage may contribute to this limitation to some extent. ${ }^{8}$ Many researchers have studied cell transplantation and/or the administration of many kinds of growth factors into cartilage defects. ${ }^{9-13}$ Some cell transplantation trials have shown success with respect to cartilage repair and have been subsequently tested in the clinical setting. ${ }^{14-16}$ However, the mechanism involved in the repair process has not yet been fully clarified. ${ }^{17}$

\footnotetext{
'Department of Orthopedic Surgery, Osaka City University Graduate School of Medicine, Osaka, Japan

${ }^{2}$ Department of Health and Sports Sciences, Mukogawa Women's University, Hyogo, Japan

${ }^{3}$ Department of Orthopedic Surgery, Itabashi Chuo Medical Center, Tokyo, Japan

${ }^{4}$ Center for Senile Degenerative Disorders (CSDD), Osaka City

University Graduate School of Medicine, Osaka, Japan

\section{Corresponding Author:}

Shigeyuki Wakitani, Department of Health and Sports Sciences, Mukogawa Women's University, 6-46 Ikebiraki, Nishinomiya 663-8558, Hyogo, Japan.

Email:wakitani@mukogawa-u.ac.jp
} 
In the past, some researchers have studied the origin of cells contributing to the tissue repair process. Shapiro et $a l .{ }^{18}$ have reported that small osteochondral defects in young rabbit cartilage were filled with mesenchymal cells within 2 weeks. They carried out titrated thymidine pulse chase experiments and could detect the signals in the bone marrow underlying the defect first and subsequently in the repair tissue, but never in the adjacent "healthy" cartilage. The authors concluded that the cells contributing to tissue repair would likely be derived from the bone marrow. Additionally, different studies suggest that cells having the potential for tissue repair could originate from other areas around the joint, including the synovium ${ }^{19-21}$ and the articular cartilage itself. ${ }^{22,23}$

These previous reports could not rule out the possibility that peripheral blood cells contribute to tissue repair, because they did not try to detect these cells. Recently, we showed that a cell population involved in tissue repair within the osteochondral defect was supplied from the peripheral blood flow using a rat parabiosis model with green fluorescent protein-transgenic and wild-type rats. ${ }^{24}$ This result could help in the development of new therapeutic strategies for osteochondral defect repair.

We hypothesized that glanulocyte colony-stimulating factor (G-CSF) might enhance the repair of osteochondral defects and produce better quality hyaline cartilage, because G-CSF increases the absolute number of peripheral blood nucleated cells. In this study, we aimed to assess the effect of G-CSF on osteochondral defect repair in rats.

\section{Materials and Methods}

\section{Animals}

Twenty-six 12-week-old male Lewis rats (Charles River Laboratories, Yokohama, Japan) were used in this study. All animals were maintained on a 24-hour light-dark cycle, with food and water available ad libitum. All experimental animal procedures were approved by and were in accordance with the regulations of the Osaka City University School of Medicine Committee on Animal Research. Twenty-six animals were randomly divided into 2 groups: a G-CSF group and a control group.

\section{Pretreatment With G-CSF}

In this study, from day 0 to day 4 , rats in the G-CSF group $(n=13)$ received a subcutaneous injection of glycosylated G-CSF (Lenograstim, Neutrogin ${ }^{\circledR}$, Chugai-Pharma, Tokyo, Japan) at $150 \mu \mathrm{g} / \mathrm{kg}$ in $500 \mu \mathrm{L}$ of phosphate-buffered saline, while control group animals $(n=13)$ received a subcutaneous injection of $500 \mu \mathrm{L}$ phosphate-buffered saline.

\section{Defect Creation}

On day 4 , the 26 rats were anesthetized by subcutaneous injections of ketamine $(50 \mathrm{mg} / \mathrm{mL}$; Sankyo, Tokyo, Japan) and xylazine $(0.2 \mathrm{mg} / \mathrm{mL}$; Bayer HealthCare, Tokyo, Japan) in a ratio of 10:3 at a dose of $1 \mathrm{~mL} / \mathrm{kg}$ body weight. The patella was dislocated laterally through a medial parapatellar approach. Subsequently, a 1.5-mm diameter and 1.0-mm deep osteochondral defect was made in the patellar groove of the femoral bone in both knees using a hand drill. The dislocated patella was returned to the original position and then sutured. All rats were allowed to move freely after surgery.

\section{Measurement of the Number of Leukocytes in the Peripheral Blood}

The number of leukocytes in the peripheral blood was measured from day 0 to day 14 using a fully automatic blood cell counter (Celltac $\alpha$, MEK- 6258; Nihon Kohden, Tokyo, Japan). Twelve rats from each group were evaluated. In addition, peripheral blood was collected from the tail of each rat.

\section{Histological Evaluation}

Following osteochondral defect surgery, 4 knees of 2 rats at 2, 4, 8, and 24 weeks, and 10 knees of 5 rats at 12 weeks were euthanized by $\mathrm{CO}_{2}$ inhalation. Both knees were collected from each animal. Following excision, the knees were fixed in 4\% paraformaldehyde (Wako Pure Chemical Industries, Ltd., Osaka, Japan) for 24 hours at $4{ }^{\circ} \mathrm{C}$, decalcified for 4 weeks, embedded in paraffin, and sectioned at a $5-\mu \mathrm{m}$ thickness. Histological analyses under light microscopy were performed using hematoxylin-eosin and toluidine blue staining.

\section{Quantitative Histological Analyses}

Quantitative histological analyses were performed with the software KIPS Ver091b (Kawasaki Heavy Industries, Ltd., Kobe, Japan) that can automatically recognize the presence of tissue and distinguish the cartilage from other tissue areas in the region of interest, based on the staining. Using toluidine blue-stained sections, we analyzed the tissue repair rate to assess the extent of defect repair. The tissue repair rate was defined as the percentage of osteochondral defects that were repaired per week. We also analyzed the area of cartilage tissue to assess the extent of cartilage repair. The cartilage repair rate was defined as the percentage of cartilage defects with metachromatic cartilage tissue that were repaired per week. The extent of cartilage repair was evaluated by the Wakitani score. ${ }^{10}$ Wakitani score was assessed by 2 observers and the average was used to analysis. 


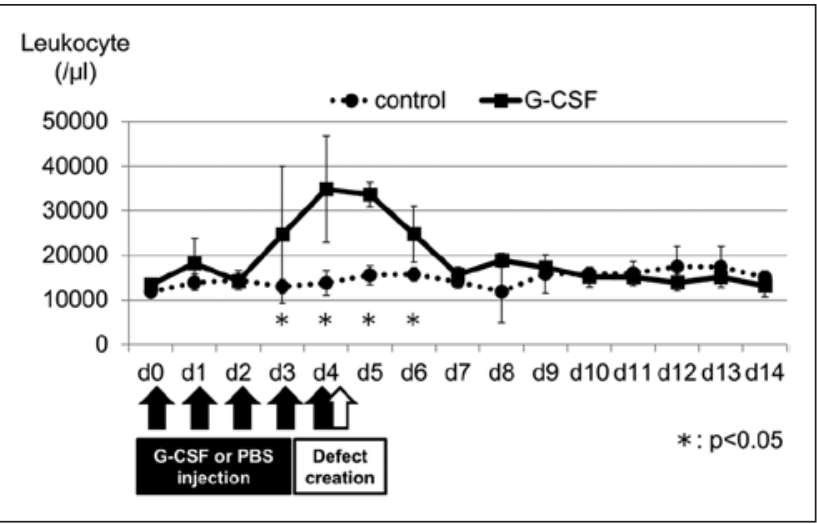

Figure I. Change in the leukocyte number. The leukocyte number in the peripheral blood was measured from day 0 to day 14 using a fully automatic blood cell counter (Celltac $\alpha$, MEK6258, Nihon Kohden, Tokyo, Japan). The number increased gradually from day 2 and reached $>30,000$ cells $/ \mu \mathrm{L}$ on day 4 . It remained elevated for about 2 days, and after day 7 , the number returned to the original level. This analysis was performed by Mann-Whitney $U$ test. Results are expressed as mean \pm standard deviation. G-CSF = granulocyte colony-stimulating factor; PBS, phosphate-buffered saline.

\section{Statistical Analysis}

The data of 2 groups were compared using the MannWhitney $U$ test. Statistical tests were considered significant at the level of $P<0.05$. All $P$ values were 2-sided.

\section{Results}

\section{The Number of Leukocytes in the Peripheral Blood}

The leukocyte number gradually increased from day 2 and reached $>30,000$ cells $/ \mu \mathrm{L}$ on day 4 (Fig. 1). This increase was maintained for about 2 days, and returned to the original level after day 7.

\section{Histological Observation}

\section{Control Groups}

Two weeks after the operation (Fig. 2A). The defects were filled with fibrous tissue, but the surface was concave. The deeper quarter of the defects were filled with a newly formed, thin cancellous bone. The superficial regions were filled with fibrous tissue with a slightly metachromatic matrix.

Four weeks after the operation (Fig. 2B). The defects were filled with thick bone in the deeper half of the defects and faintly metachromatic fibrous tissue in the superficial layer. The surface was still slightly concave.
Eight weeks after the operation (Fig. 2C). The repaired tissue showed a smooth articular surface, but the defects were filled with fibrous tissue with a faintly metachromatic cartilage-like tissue.

Twelve weeks after the operation (Fig. 2D). The subchondral bone had been almost completely reformed. The defects became covered with cartilage-like tissue with round cells and a metachromatic matrix. The repaired surface remained smooth.

Twenty-four weeks after the operation (Fig. 2E). Subchondral bone had been almost entirely reformed, and a cartilage-like tissue had been deposited on the surface. Metachromatic staining was observed in the surface layer, and the repaired surface remained smooth. The repaired tissue appeared to be fibrous rather than hyaline cartilage.

Granulocyte Colony-Stimulating Factor Groups. The repair processes in the G-CSF group were almost the same as those of the control group, except for the following points.

Two weeks after the operation (Fig. 2F). The defects were filled with more boney tissue than in the control group. The surface was slightly concave.

Four weeks after the operation (Fig. 2G). The thickness of the fibrous tissue of the deeper zone was reduced, and new bone filled this defect region. Subchondral bone formation was superior to that seen in the control group. More cartilage cells were seen in the middle region, and the superficial regions were filled with a metachromatic matrix. The surface was raised and smooth.

Eight weeks after the operation (Fig. 2H). The subchondral bone had been almost reformed, the surface was flat, and the thickness of the cartilage layer was increased. Metachromatic cartilage cells were seen in the middle layer.

Twelve weeks after the operation (Fig. 2I). The subchondral bone had been almost completely reformed.

Twenty-four weeks after the operation (Fig. 2J). The subchondral bone had been completely reformed. The defects were filled in with cartilage, which was slightly thinner than that at 12 weeks.

In the time course of the histological observations (toluidine blue staining), the repair processes in the G-CSF group were better than those in the control group, particularly at the early time points.

\section{Quantitative Histological Analyses}

No significant difference in the tissue repair rate between the 2 groups was observed 2 weeks after surgery (Fig. 3A). 

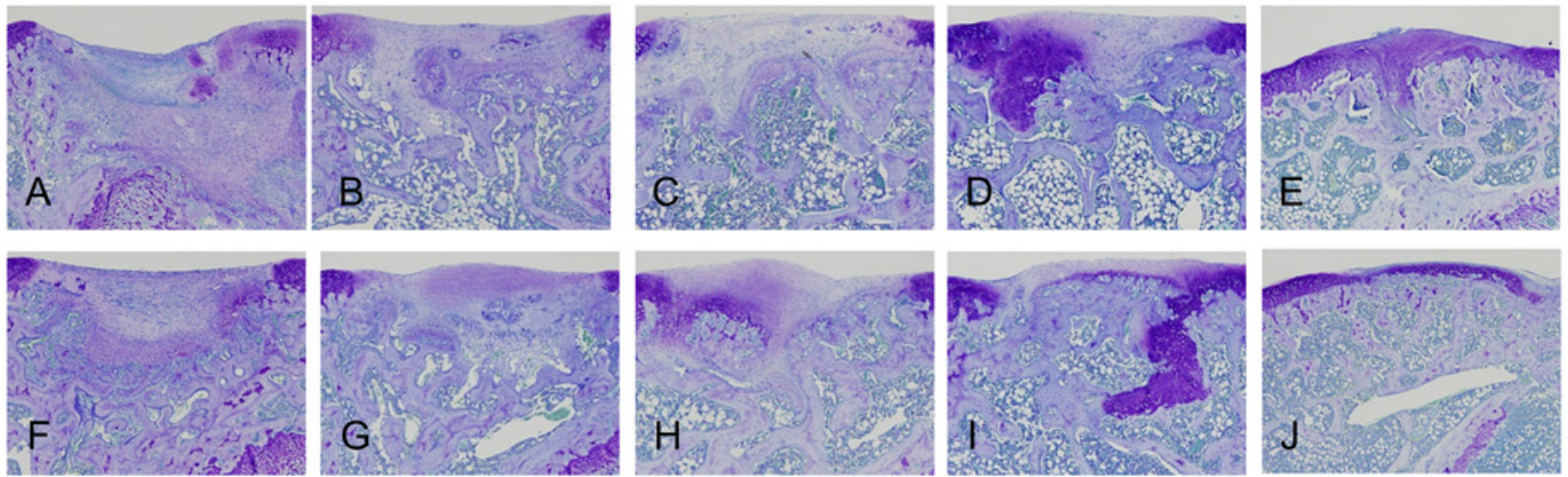

Figure 2. Histological findings. Time course of the histological observations: (A) control group at 2 weeks, (B) control group at 4 weeks, (C) control group at 8 weeks, (D) control group at 12 weeks, (E) control group at 24 weeks, (F) G-CSF group at 2 weeks, (G) G-CSF group at 4 weeks, (H) G-CSF group at 8 weeks, (I) G-CSF group at 12 weeks, and (J) G-CSF group at 24 weeks (toluidine blue staining). The repair processes in the G-CSF group were better than that in the control group at the early time points. Two weeks following the osteochondral surgery in the G-CSF group, the defects were more occupied with minimally cellular fibrous tissue and at 4 weeks the defects were more occupied with fibrous and bony tissue. However, after 8 weeks, the difference in the composition of repaired tissue in the 2 groups was less evident than that in the earlier phases. G-CSF = granulocyte colony-stimulating factor.

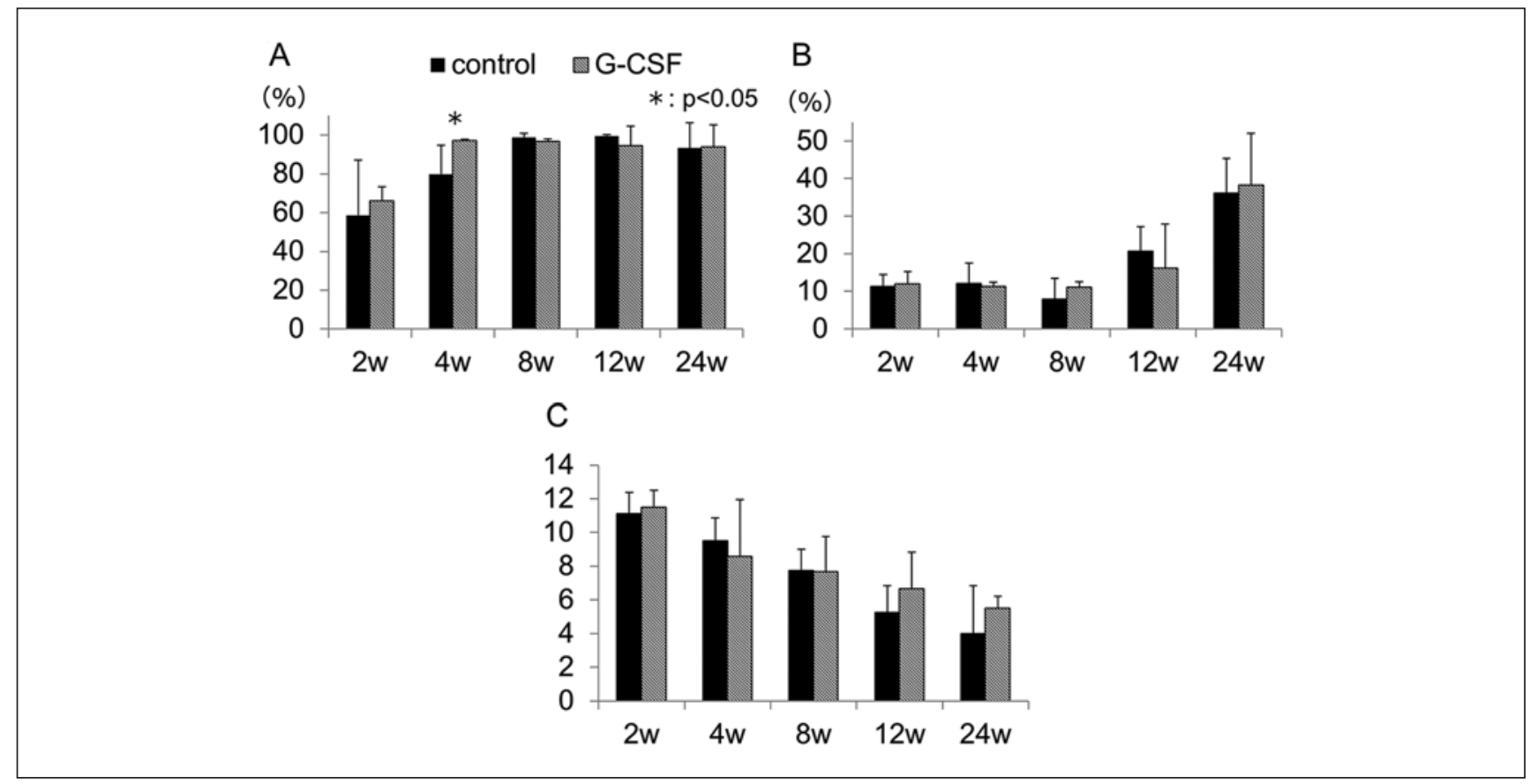

Figure 3. Quantification of various histological parameters. (A) Tissue repair rate. A significant difference was not observed between the 2 groups 2 weeks after surgery. However, at 4 weeks, the tissue repair rate was significantly higher in the granulocyte colonystimulating factor (G-CSF) group. This analysis was performed by Mann-Whitney $U$ test. (B) Cartilage repair rate. (C) Modified Wakitani score. There was no significant difference between the 2 groups at any of the time points. This analysis was performed by Mann-Whitney $U$ test. Results are expressed as mean \pm standard deviation.

However, 4 weeks after surgery, the tissue repair rate was significantly higher in the G-CSF group.

Cartilage repair rate (Fig. 3B) and modified Wakitani score (Fig. 3C) were not significantly different between the 2 groups.

\section{Discussion}

In this study, the tissue repair rate was significantly higher (the surfaces were flat or slightly convex, but not concave) in the G-CSF group 4 weeks after surgery. Histological 
analysis showed that the tissues of the G-CSF group rats filled better and started filling earlier, suggesting that G-CSF could promote the repair of osteochondral defects by increasing the number of peripheral blood nucleated cells. To our knowledge, this study is the first to demonstrate that systemic administration of agents may be effective in promoting focal osteochondral defect repair in vivo; moreover, G-CSF could be one candidate for an agent.

Recently, some options are available for the treatment of osteochondral defects, including bone marrow stimulation, cartilage transplantation, ${ }^{25}$ autologous chondrocyte implantation, ${ }^{15,26-28}$ or the combination of some of these treatments. The bone marrow stimulation technique repairs the osteochondral defect with fibrocartilage, which is known to be biochemically and biomechanically different from normal hyaline cartilage, and tissue degeneration is a concern in the reparative tissues over a long period of time. ${ }^{29,30}$ Nevertheless, this technique is still standard for articular cartilage repair methods because of low invasiveness, low cost, and ease of performance.

We previously reported the cells from the peripheral blood would contribute to tissue repair by using a rat parabiosis model with green fluorescent protein-transgenic and wildtype rats. ${ }^{24}$ Our results supported the possibility that the peripheral blood cells also contribute to osteochondral repair. Therefore, the systemic administration of any agent that enhances peripheral blood cell numbers during osteochondral repair appears to be a promising therapeutic approach.

Granulocyte colony-stimulating factor is a major extracellular regulator of hemopoiesis and the innate immune system. Named for its relatively specific stimulation of the growth of neutrophil progenitor cells in vitro in semisolid cultures, ${ }^{31,32}$ G-CSF influences the survival, proliferation, and differentiation of all cells in the neutrophil lineage, from hemopoietic stem cells to mature neutrophils. Furthermore, G-CSF influences the function of mature neutrophils. ${ }^{33}$ In addition, it is possible that the mesenchymal stem cells were increased at the same time, increasing the number of neutrophils in bone marrow and stimulating the release of mesenchymal stem cells. These roles help explain the rapid uptake of G-CSF into clinical medicine as a drug that increases the production of neutrophils in patients with chemotherapy-induced neutropenia. ${ }^{34}$ In the cardiac field, the usefulness of G-CSF for tissue repair has been shown in some papers. Janssens reported that the prospective randomized multicenter studies in AMI patients at increased risk for developing heart failure are required to evaluate whether or not the observed changes in remodeling parameters following early G-CSF treatment translate into a better clinical outcome and survival. ${ }^{35}$ Moreover, Shim et al. ${ }^{36}$ reported that cardiac repair by G-CSF therapy is a safe therapeutic approach. Therefore, we administered G-CSF through the systemic route to test if it improved cartilage repair in osteochondral defects. On the other hand, it was reported that G-CSF may play as pro-inflammatory role. This process was found during a sustained and prolonged release in the joint environment, as observed during the onset of inflammatory arthritis, along with a critical role of synovial tissue $\mathrm{e}^{37}$ and neutrophils in the genesis of the degenerative changes observed in the articular cartilage. ${ }^{38}$ The possibility that G-CSF might influence negatively to tissue repair was not completely denied.

We observed that the G-CSF group showed better tissue filling and started earlier, whereas the absolute number of peripheral blood nucleated cells was raised beyond the normal level only on days 3 to 7 after injection of G-CSF. This result suggests that the peripheral blood nucleated cells enhanced by G-CSF contribute to the early phase of the healing process for osteochondral defects. Shapiro et al. ${ }^{18}$ reported the origin and differentiation of cells for the repair of full-thickness defects of articular cartilage were studied histologically in rabbits. In the first few days, fibrous arcades were established across the defect, and this served to orient mesenchymal cell ingrowth. ${ }^{18}$ At 4 to 8 weeks, the repaired cartilage took on a true hyaline appearance. In this study, G-CSF was subcutaneously injected into rats from day 0 to day 4 , for a total of 5 days. The peripheral nucleated cells were raised 3 days after the first injection, reached more than 30,000 cells/ $\mu \mathrm{L}$ at day 4 and 5 , and returned to the normal levels 7 days after the first injection. Although we observed the tissue repair rate except for 4 weeks after surgery, cartilage repair rate and modified Wakitani score were not significantly different between the 2 groups. We consider that the duration of raised the number of leukocyte was not long enough. Improved cartilage repair might be expected if the higher number of peripheral blood cells could be maintained for a longer time than that in our experiment. Peripheral blood cells are considered to produce cytokines. ${ }^{39}$ However, while we only examined the number of peripheral blood cells, cytokine production by these cells was not evaluated. It is possible that not only the cells directly, but humoral factors also may be involved in repair in the early phases. Further studies would be required to fully elucidate the effect of G-CSF on osteochondral repair.

\section{Conclusions}

In this study, we aimed to assess the effect of G-CSF on osteochondral defect repair in a rat experimental model. Based on the histological findings, the G-CSF group showed improved tissue filling and started earlier. These results suggest that G-CSF could act as a promoting agent in the repair of osteochondral defects by increasing the number of peripheral blood nucleated cells.

\section{Acknowledgments and Funding}

The authors are grateful to Kanako Hata, Osaka City University Graduate School of Medicine, Japan, for providing us with 
technical assistance. The author(s) received no financial support for the research, authorship, and/or publication of this article.

\section{Declaration of Conflicting Interests}

The author(s) declared no potential conflicts of interest with respect to the research, authorship, and/or publication of this article.

\section{Ethical Approval}

This study was approved by our institutional review board.

\section{References}

1. Curl WW, Krome J, Gordon ES, Rushing J, Smith BP, Poehling GG. Cartilage injuries: a review of 31,516 knee arthroscopies. Arthroscopy. 1997;13:456-60.

2. Hjelle K, Solheim E, Strand T, Muri R, Brittberg M. Articular cartilage defects in 1,000 knee arthroscopies. Arthroscopy. 2002;18:730-4.

3. Ding C, Cicuttini F, Scott F, Stankovich J, Cooley H, Jones $\mathrm{G}$. The genetic contribution and relevance of knee cartilage defects: case-control and sib-pair studies. J Rheumatol. 2005;32:1937-42.

4. Ding C, Cicuttini F, Jones G. How important is MRI for detecting early osteoarthritis? Nat Clin Pract Rheumatol. 2008;4:4-5.

5. Mankin HJ. The response of articular cartilage to mechanical injury. J Bone Joint Surg Am. 1982;64:460-6.

6. Convery FR, Akeson WH, Keown GH. The repair of large osteochondral defects. An experimental study in horses. Clin Orthop Relat Res. 1972;82:253-62.

7. Lietman SA, Miyamoto S, Brown PR, Inoue N, Reddi AH. The temporal sequence of spontaneous repair of osteochondral defects in the knees of rabbits is dependent on the geometry of the defect. J Bone Joint Surg Br. 2002;84:600-6.

8. Urist MR, Adams T. Cartilage or bone induction by articular cartilage. Observations with radioisotope labelling techniques. J Bone Joint Surg Br. 1968;50:198-215.

9. Nawata M, Wakitani S, Nakaya H, Tanigami A, Seki T, Nakamura Y, et al. Use of bone morphogenetic protein 2 and diffusion chambers to engineer cartilage tissue for the repair of defects in articular cartilage. Arthritis Rheum. 2005;52:155-63.

10. Wakitani S, Goto T, Pineda SJ, Young RG, Mansour JM, Caplan AI, et al. Mesenchymal cell-based repair of large, fullthickness defects of articular cartilage. J Bone Joint Surg Am. 1994;76:579-92.

11. Wei JP, Nawata M, Wakitani S, Kametani K, Ota M, Toda A, et al. Human amniotic mesenchymal cells differentiate into chondrocytes. Cloning Stem Cells. 2009;11:19-26.

12. Nishimoto N, Yoshizaki K, Miyasaka N, et al. Treatment of rheumatoid arthritis with humanized anti-interleukin-6 receptor antibody: a multicenter, double-blind, placebo-controlled trial. Arthritis Rheum. 2004;50:1761-9.

13. Zscharnack M, Hepp P, Richter R, Aigner T, Schulz R, Somerson J, et al. Repair of chronic osteochondral defects using predifferentiated mesenchymal stem cells in an ovine model. Am J Sports Med. 2010;38:1857-69.
14. Wakitani S, Mitsuoka T, Nakamura N, Toritsuka Y, Nakamura Y, Horibe S. Autologous bone marrow stromal cell transplantation for repair of full-thickness articular cartilage defects in human patellae: two case reports. Cell Transplant. 2004;13:595-600.

15. Wakitani S, Nawata M, Tensho K, Okabe T, Machida H, Ohgushi H. Repair of articular cartilage defects in the patellofemoral joint with autologous bone marrow mesenchymal cell transplantation: three case reports involving nine defects in five knees. J Tissue Eng Regen Med. 2007;1:74-9.

16. Wakitani S, Okabe T, Horibe S, Mitsuoka T, Saito M, Koyama T, et al. Safety of autologous bone marrow-derived mesenchymal stem cell transplantation for cartilage repair in 41 patients with 45 joints followed for up to 11 years and 5 months. J Tissue Eng Regen Med. 2011;5:146-50.

17. Dell'accio F, Vincent TL. Joint surface defects: clinical course and cellular response in spontaneous and experimental lesions. Eur Cell Mater. 2010;20:210-7.

18. Shapiro F, Koide S, Glimcher MJ. Cell origin and differentiation in the repair of full-thickness defects of articular cartilage. J Bone Joint Surg Am. 1993;75:532-53.

19. De Bari C, Dell'Accio F, Luyten FP. Human periosteumderived cells maintain phenotypic stability and chondrogenic potential throughout expansion regardless of donor age. Arthritis Rheum. 2001;44:85-95.

20. De Bari C, Dell'Accio F, Tylzanowski P, Luyten FP. Multipotent mesenchymal stem cells from adult human synovial membrane. Arthritis Rheum. 2001;44:1928-42.

21. Hunziker EB, Rosenberg LC. Repair of partial-thickness defects in articular cartilage: cell recruitment from the synovial membrane. J Bone Joint Surg Am. 1996;78:721-33.

22. Dell'Accio F, De Bari C, Luyten FP. Microenvironment and phenotypic stability specify tissue formation by human articular cartilage-derived cells in vivo. Exp Cell Res. 2003;287:16-27.

23. Dowthwaite GP, Bishop JC, Redman SN, Khan IM, Rooney P, Evans DJ, et al. The surface of articular cartilage contains a progenitor cell population. J Cell Sci. 2004;117:889-97.

24. Okano T, Wakitani S, Okabe T, Takahashi M, Koike T, Nakamura H. Nucleated cells circulating in the peripheral blood contribute to the repair of osteochondral defects only in the early phase of healing. J Tissue Eng Regen Med. Epub 2012 Jun 4.

25. Matsusue Y, Yamamuro T, Hama H. Arthroscopic multiple osteochondral transplantation to the chondral defect in the knee associated with anterior cruciate ligament disruption. Arthroscopy. 1993;9:318-21.

26. Grande DA, Pitman MI, Peterson L, Menche D, Klein M. The repair of experimentally produced defects in rabbit articular cartilage by autologous chondrocyte transplantation. J Orthop Res. 1989;7:208-18.

27. Peterson L, Minas T, Brittberg M, Nilsson A, Sjögren-Jansson E, Lindahl A. Two- to 9-year outcome after autologous chondrocyte transplantation of the knee. Clin Orthop Relat Res. 2000;(374):212-34.

28. Wakitani S, Imoto K, Yamamoto T, Saito M, Murata N, Yoneda M. Human autologous culture expanded bone marrow mesenchymal cell transplantation for repair of cartilage defects in osteoarthritic knees. Osteoarthritis Cartilage. 2002;10:199-206. 
29. Matsumoto T, Okabe T, Ikawa T, Iida T, Yasuda H, Nakamura $\mathrm{H}$, et al. Articular cartilage repair with autologous bone marrow mesenchymal cells. J Cell Physiol. 2010;225:291-5.

30. Wakitani S, Kawaguchi A, Tokuhara Y, Takaoka K. Present status of and future direction for articular cartilage repair. J Bone Miner Metab. 2008;26:115-22.

31. Metcalf D, Nicola NA. Proliferative effects of purified granulocyte colony-stimulating factor (G-CSF) on normal mouse hemopoietic cells. J Cell Physiol. 1983;116:198-206.

32. Burgess AW, Metcalf $D$. The nature and action of granulocytemacrophage colony stimulating factors. Blood. 1980;56:947-58.

33. Basu S, Dunn A, Ward A. G-CSF: Function and modes of action (review). Int J Mol Med. 2002;10:3-10.

34. Hübel K, Engert A. Clinical applications of granulocyte colony-stimulating factor: an update and summary. Ann Hematol. 2003;82:207-13.

35. Janssens SP. Granulocyte colony-stimulating factor in cardiac repair: time for a reappraisal? Eur J Heart Fail. 2010;12:1021-3.
36. Shim W, Mehta A, Lim SY, Zhang G, Lim CH, Chua T, et al. G-CSF for stem cell therapy in acute myocardial infarction: friend or foe? Cardiovasc Res. 2011;89:20-30.

37. van Holten J, Reedquist K, Sattonet-Roche P, Smeets TJ, Plater-Zyberk C, Vervoordeldonk MJ, et al. Treatment with recombinant interferon- $\beta$ reduces inflammation and slows cartilage destruction in the collagen-induced arthritis model of rheumatoid arthritis. Arthritis Res Ther. 2004;6:R239-49.

38. Katano M, Okamoto K, Arito M, Kawakami Y, Kurokawa MS, Suematsu N, et al. Implication of granulocytemacrophage colony-stimulating factor induced neutrophil gelatinase-associated lipocalin in pathogenesis of rheumatoid arthritis revealed by proteome analysis. Arthritis Res Ther. 2009;11:R3.

39. Imanishi $\mathrm{Y}$, Miyagawa S, Saito A, Kitagawa-Sakakida S, Sawa Y. Allogenic skeletal myoblast transplantation in acute myocardial infarction model rats. Transplantation. 2011;91:425-31. 